\title{
2-咪唑啉类化合物的合成及应用研究新进展
}

\author{
周葆悦张金李向南厍梦尧张劲 \\ 李剑利* 史 真
}

(西北大学化学与材料科学学院 合成与天然功能分子化学教育部重点实验室 西安 710069)

\begin{abstract}
摘要 2-咪唑啉是一类重要的五元含氮杂环化合物, 基于其良好的反应性及生物活性在药物化学、天然产物化学、防 腐缓蚀及催化等领域应用广泛，一直以来此类化合物的合成和应用拓展倍受关注. 综述了近年来 2-咪唑啉类化合物合 成及其应用研究的新进展.
\end{abstract}

关键词 2-咪夾啉; 合成方法; 应用

\section{Progress in the Synthesis and Applications of 2-Imidazolines}

\author{
Zhou, Baoyue Zhang, Jin Li, Xiangnan She, Mengyao \\ Zhang, Jing Li, Jianli* Shi, Zhen \\ (Key Laboratory of Synthetic and Natural Functional Molecular Chemistry, Ministry of Education, \\ College of Chemistry \& Materials Science, Northwest University, Xi'an 710069)
}

\begin{abstract}
As an important class of heterocyclic scaffolds, 2-imidazolines and their derivatives are an important class of molecules. Based on their good reactivity and biological activity, they are widely used in the field of drugs and pharmaceuticals, natural product chemistry, synthetic organic chemistry, coordination chemistry, anticorrosion and homogeneous catalysis. Significant effort has been devoted to the synthesis and applications of such compounds. This review provides a summary of recently developed methods for the preparation of 2-imidazolines, and their latest developments of applications.

Keywords 2-imidazolines; synthesis method; application
\end{abstract}

含氮五元杂环骨架的构建及广泛应用，展现了丰富 美妙的化学合成魅力. 咪唑啉是五元含氮杂环化合物中 重要的代表物, 此结构单元多为药物、天然产物、金属 有机催化剂以及缓蚀剂的关键结构片段，咪唑啉杂环结 构单元的合成及构建一直以来是杂环化学研究领域的 热点 ${ }^{[1]}$. 咪唑啉因其双键位置的不同, 可分为 2-咪唑啉、 3-咪唑啉及 4-咪唑啉. 2-咪唑啉及其衍生物因特殊的结 构特征及优异的反应活性, 使其在有机化学领域具有极 高的研究意义和应用价值.

2-咪唑啉类化合物的多类型合成方法被大量报道. 2009 年, $\mathrm{Crouch}^{[2]}$ 及 $\mathrm{Du}$ 等 ${ }^{[3]}$ 曾从不同合成方法归类角度 对 2-咪唑啉类化合物的合成方法进行总结, 并侧重介绍 了其作为配体在催化合成领域中的应用进展. 近年来,
2-咪唑啉类化合物的研究依然活跃, 尤其是结构新颖的 衍生物合成报道大量涌现. 本文在此基础上，结合我们 课题组咪唑啉系列五元含氮杂环化合物的合成研究, 综 述了近年来基于合成底物不同的 2-咪唑啉及其衍生物 的合成新进展, 并结合实例简述其广阔的应用前景.

\section{2-咪唑啉类化合物的合成方法}

\section{1 以腈与二胺为底物合成}

腈与乙二胺的盐类化合物直接环合制备 2-咪唑啉 的方法(Eq. 1), 因耗时较长, 具有很大局限性 ${ }^{[4]} .1981$

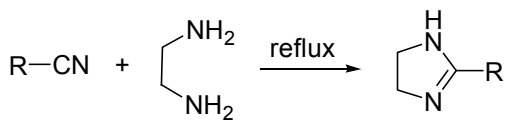

\footnotetext{
*E-mail: lijianli@nwu.edu.cn

Received July 30, 2012; revised October 17, 2012; published online October 24, 2012.

Project supported by the National Natural Science Foundation of China (No. 20972124), the Shaanxi Science and Technology Co-ordination Innovation Engineering Project (No. 2011K12-77), the Xi'an City Science and Technology Project (No. CXY1123-1).

国家自然科学基金(No. 20972124)、陕西省科技统筹创新工程计划项目(No. 2011K12-77)、西安市科技计划(No. CXY1123-1)资助项目.
} 
年, Levesque 等 ${ }^{[5]}$ 首次向反应体系中引入 $\mathrm{H}_{2} \mathrm{~S}$ 发现底物 的利用率显著提高. 此报道虽未能彻底减少反应耗时, 但对反应机理的探究具有指导性(Scheme 1). 2004 年, Dash 等 ${ }^{[6]}$ 报道了硫代乙酰胺催化的 2-咪唑啉合成方法, 反应经 3 3.5 h 关环得到多种取代 2-咪唑啉化合物, 且 产率较高. 基于硫粉可与腈反应生成硫代酰胺, 2006 年, Mirkhani 等 ${ }^{[7]}$ 对合成方法进行了扩展, 实现了在超声波 促进下较短的时间内制备 2-咪唑啉.

2007 年, Mirkhani 等 ${ }^{[8]}$ 发现廉价、高活性、易制备 且低毒性的 $\mathrm{ZrOCl}_{2} \bullet 8 \mathrm{H}_{2} \mathrm{O}$ 可用作反应的催化剂, 该法可 在加热、微波及超声波中分别进行, 微波及超声波的协 助不但提升了反应产率, 亦可减少副产物的生成, 同时, $\mathrm{ZrOCl}_{2} \cdot 8 \mathrm{H}_{2} \mathrm{O}$ 经简单处理可重复利用并仍保持良好活 性. 2008 年, Mahammadpoor-Baltork 等 ${ }^{[9,10]}$ 先后以高活 性、易制备、可重复利用的硅硫酸(SSA)及磷铇酸(TPA) 为催化剂合成了噁唑啉、咪唑啉及噻唑啉, 并提出了这 类金属催化剂的作用机理(Scheme 2).

2009 年, Shaabani 等 ${ }^{[11]}$ 报道了一种以纤维素硫酸 (CSA)为催化剂合成 2-咪唑啉的方法, 此合成法催化剂
用量少、反应耗时短且合成效率高, 不仅适用于催化咪 唑啉的合成，还可用于噻唑啉和噁唑啉的制备. 2010 年 及 2011 年, Nase-Esfahani 等 ${ }^{[12,13]}$ 先后提出了在无溶剂条 件下，以硅铇酸 $(\mathrm{STA})$ 及对甲苯磺酸 $(p-\mathrm{TSA})$ 为催化剂合 成多种 2-味唑啉类化合物的方法. 这两种合成方法操作 简便易控制、催化剂活性高且稳定性佳(Eq. 2).

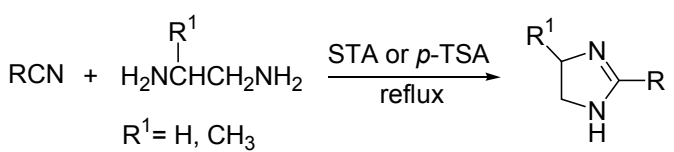

本课题组多年来曾对咪唑啉等五元含氮杂环化合 物的合成法进行过系统的研究, 不但对真空法合成咪唑 啉进行了有益的改进及应用深入 ${ }^{[14]}$, 并且意外地发现 吲哚乙酸铜可用于催化腈与二胺合成 2-咪唑啉 ${ }^{[15]}$, 进而 拓展至苯甲酸铜催化噻唑啉及噁唑啉的合成 ${ }^{[16]}$ (Scheme 3). 研究发现, 吲哚乙酸铜催化合成 2-咪唑啉 法若在微波辅助下， $20 \mathrm{~min}$ 内即可实现，且产率较高， 此反应体系适用于多种芳香腈, 其突出特点是催化剂稳 定、活性高且可重复使用. 这也是首例利用调控植物生

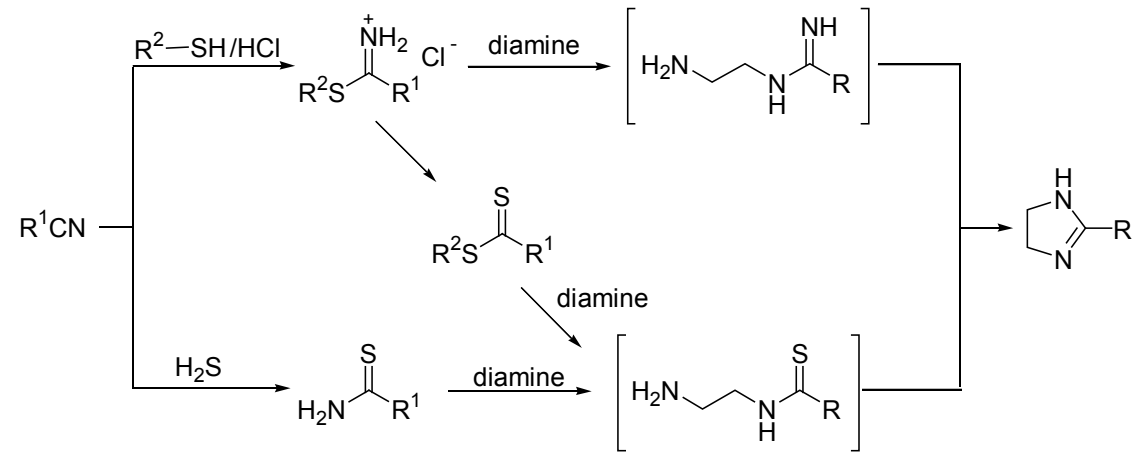

Scheme 1
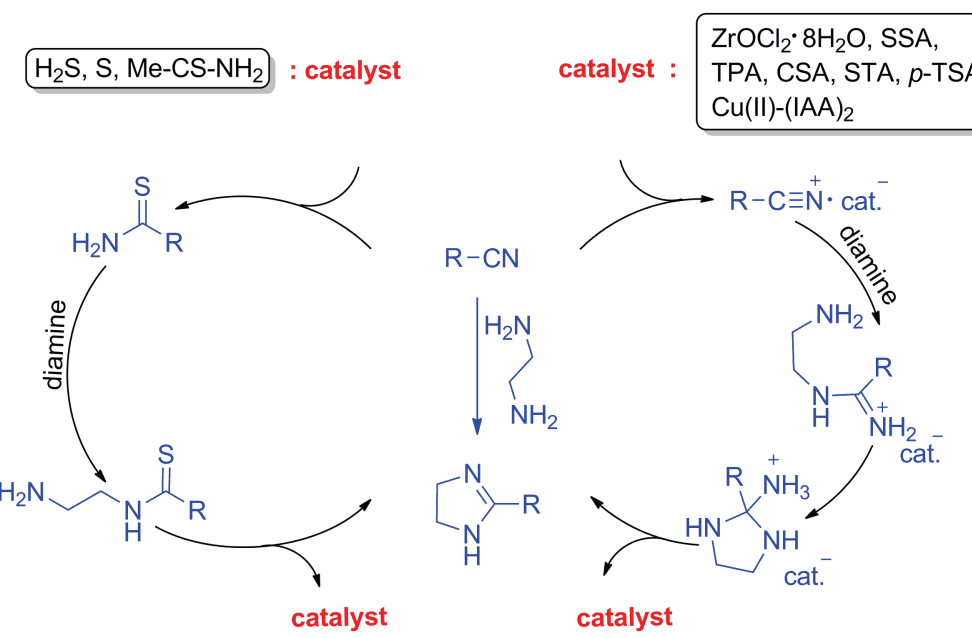

Scheme 2 


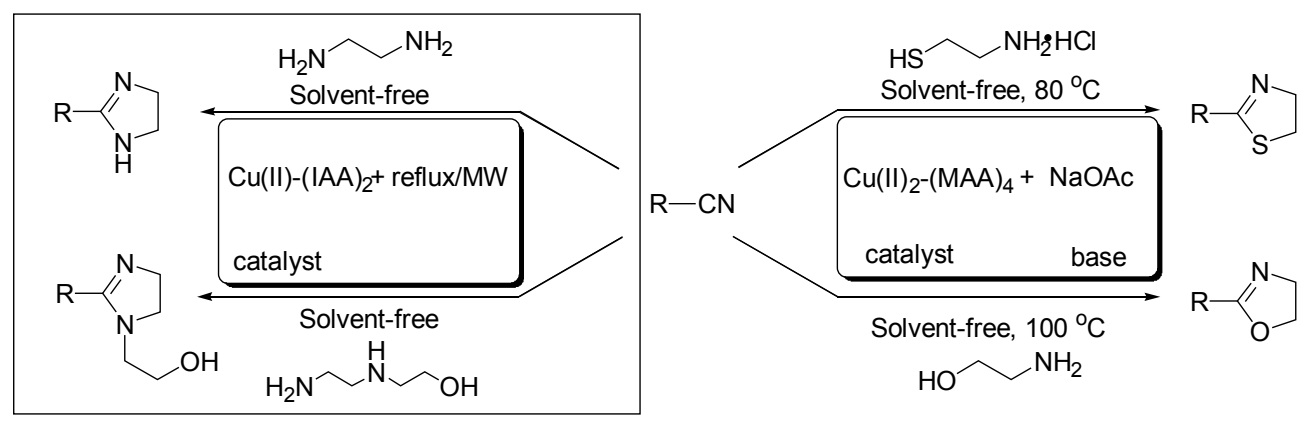

$\mathrm{R}=$ Aryl, Pyridyl, Non-conjugated groups

Scheme 3

长发育的激素吲哚乙酸作为此类有机反应催化剂配体 的合成方法, 获得极优异的催化调控效应.

\section{2 以腈与唁丙啶为底物合成}

呋丙啶是一类被广泛用于有机合成的叔胺, 其典型 的反应是扩环过程, 此类化合物的丰富活性取决于氮原 子上取代基的结构特性: 氮原子上带有吸电子取代基的 呋丙啶易与亲核试剂作用开环; 而含有给电子基团的呋 丙啶则需在路易斯酸的协助下才能与亲核试剂反应. 1973 年, Nozaki 等 ${ }^{[17]}$ 首次提出可通过腈与带有吸电子基 团的呋丙啶 1 反应制备 2-咪唑啉类化合物 2 (Eq. 3).

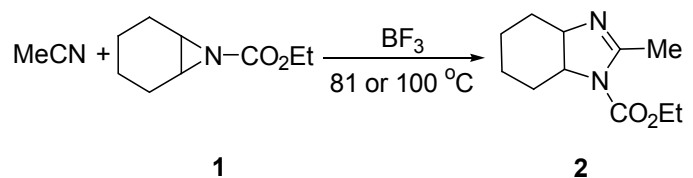

2004 年, Concellón 等 ${ }^{[18]}$ 对此工作进行了拓展, 提出 $\mathrm{BF}_{3} \cdot \mathrm{OEt}_{2}$ 催化多种腈与鲐丙啶反应制备 2-咪唑啉 (Scheme 4). 实验发现, 呋丙啶的开环遵循一定的立体 选择性, 因此合成得到的 2-咪唑啉类物质具有较好的光 学纯度. Concellón 认为其催化机制首先是 $\mathrm{BF}_{3} \cdot \mathrm{OEt}_{2}$ 进 攻氮原子使环断裂, 随后腈作为亲核试剂参与反应并生 成中间体, 最终关环得到 2-咪唑啉及其功能衍生物.

2007 年, Gandhi 等 ${ }^{[19]}$ 利用[3+2]环加成反应成功设 计合成了多种 2-咪唑啉类衍生物(Eq. 4), 此反应在 10 $\min$ 内即可彻底完成且 $\mathrm{BF}_{3} \cdot \mathrm{OEt}_{2}$ 及 $\mathrm{Et}_{3} \mathrm{OBF}_{4}$ 的催化效果 显著. 与 Concellón 方法不同的是, 产物的空间结构由 合成底物与催化剂性质共同决定, 并不遵循特定的规 则.

2011 年, $\mathrm{Li}$ 等 ${ }^{[20]}$ 尝试拓展更多的路易斯酸作为这类 反应的催化剂, 发现 $\mathrm{Bi}(\mathrm{OTf})_{3}$ 的效果突出(Scheme 5). 在对反应溶剂、温度及时间等条件的探索中, $\mathrm{Li}$ 发现溶 剂及温度对催化效果有决定性影响, 当以乙腈为溶剂在 室温条件下进行时, 催化剂可保持最佳活性且无副反 应. 同年, 魏文珑等 ${ }^{[21]}$ 对以腈与吅丙啶为原料通过 $[3+$
2]环加成反应制备 2-咪唑啉类化合物的方法进行了总 结.

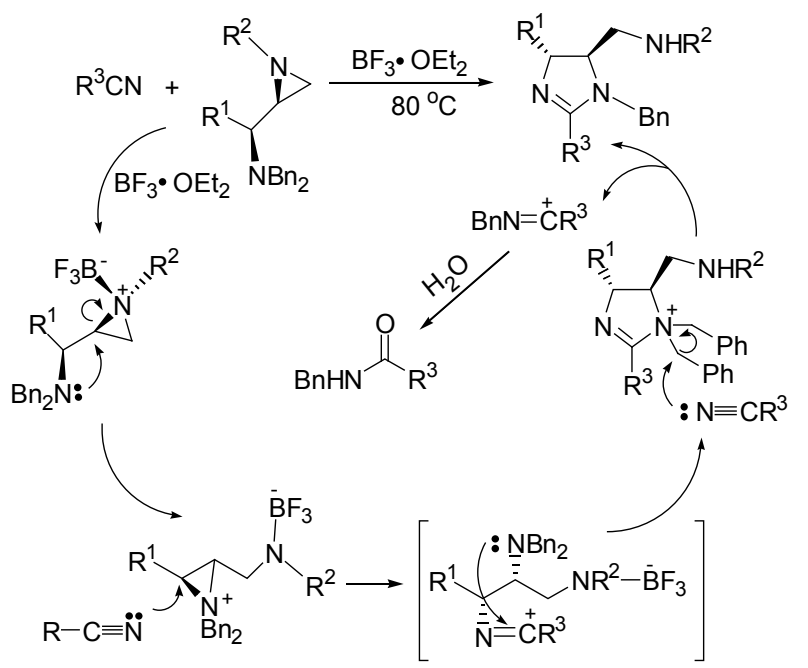

Scheme 4
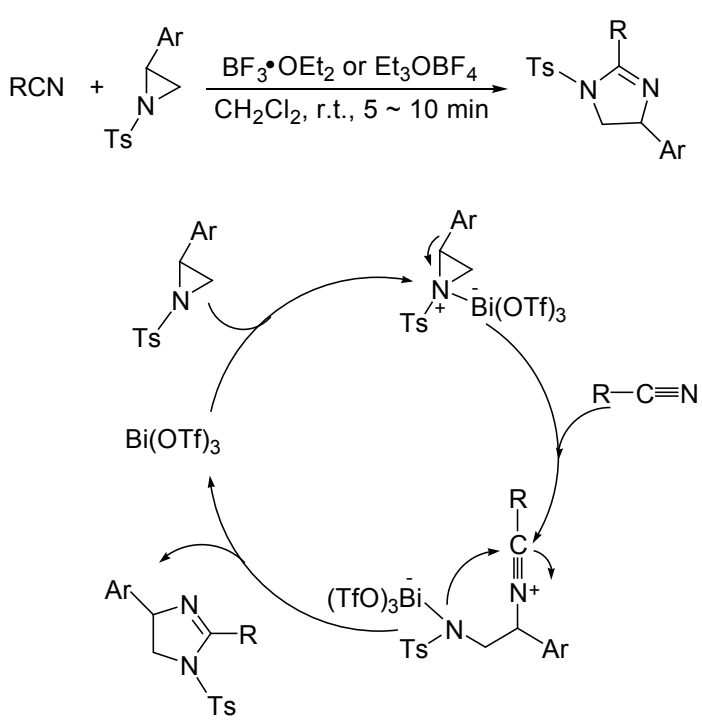

Scheme 5

Benito-Garagorri 等 ${ }^{[22]}$ 曾于 2006 年使用较少见的一 价铜盐配合物为催化剂, 成功地以腈 $\mathbf{3}$ 与亚胺 $\mathbf{4}$ 为底物 
合成了 2-咪唑啉类衍生物 5 (Eq. 5).

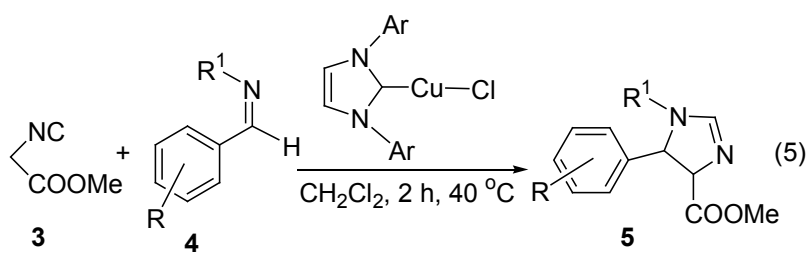

2012 年, Nakamura 等 ${ }^{[23]}$ 首次报道了以手性硫脲为 催化剂的制备多取代 2-咪唑啉类化合物的方法(Eq. 6). 该方法通过腈与 $N$-磺酰基亚胺的 Mannich 反应实现, 不 但高效且产物纯度高.

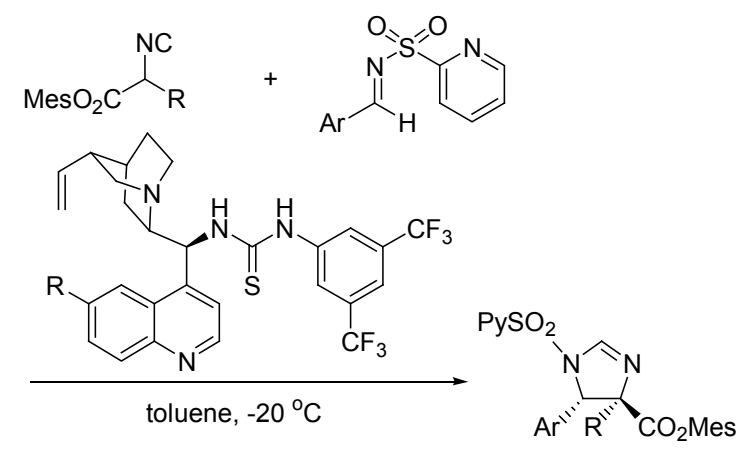

\section{3 以羫酸及其衍生物为底物合成}

羧酸及其衍生物也常被用作合成 2-咪唑啉的基础 合成底物. 胺与羧酸或酯混合加热即可生成 2-咪唑啉, 但苛刻的反应条件限制了其广泛应用. 1981 年，Neef 等 ${ }^{[24]}$ 发现向上述反应体系中加入少量 $\mathrm{Me}_{3} \mathrm{Al}$ 时, 反应较 易进行且产率可达 96\% (Scheme 6).

$$
\begin{aligned}
& \mathrm{RCOOR}^{1}+\mathrm{NH}_{2} \mathrm{CH}_{2} \mathrm{CH}_{2} \mathrm{NH}_{2} \underset{\text { reflux }}{\stackrel{\left(\mathrm{CH}_{3}\right)_{3} \mathrm{Al}}{\longrightarrow}}\left[\mathrm{R} \underset{\mathrm{HN}-\mathrm{CH}_{2}}{\stackrel{\mathrm{C}}{\mathrm{CH}_{2}}} \begin{array}{c}
\mathrm{NH}_{2} \\
\\
\end{array}\right] \\
& \mathrm{R}-\mathrm{K}_{\mathrm{N}}^{\mathrm{N}}
\end{aligned}
$$

\section{Scheme 6}

Aleksandrov 等 ${ }^{[25]}$ 同样致力于以羧酸及其衍生物为 底物的合成方法的改进. 2009 年, Aleksandrov 将羧酸及 $50 \% \sim 57 \%$ 的乙二胺于乙烯基乙二醇中混合, 并以苯磺 酸 (PTSA) 为催化剂进行反应, 获得了良好的收率 (Scheme 7).

Boland 等 ${ }^{[26]}$ 进一步研究发现, 差基酰胺经两次氯 代重排后生成的中间体 $\mathbf{6}$ 具有极高的活性, 能快速与胺 作用关环生成 2-咪唑啉类化合物 7, 且得到的咪唑啉具 有手性多被用作配体(Scheme 8).

You 等 ${ }^{[27]}$ 以 $N$-酰化- $\beta$-氨基- $\alpha$-氨基酯 8 为合成底物

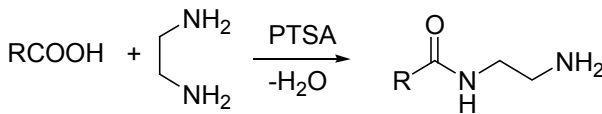

$$
\begin{aligned}
& \stackrel{-\mathrm{H}_{2} \mathrm{O}}{\mathrm{Heat}}
\end{aligned}
$$

Scheme 7
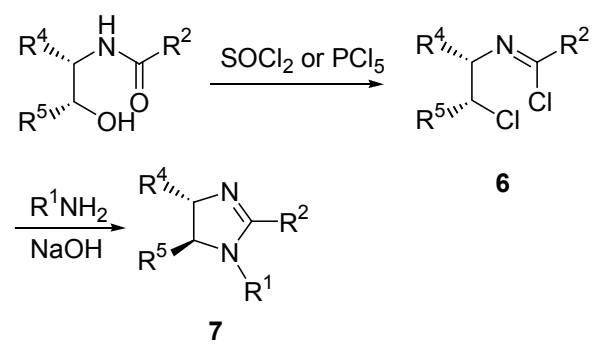

6

\section{Scheme 8}

制备了多种含羧酸酯结构的 2-咪唑啉类化合物 9 (Eq.7). 这些 2-咪唑啉衍生物很好地保留了合成底物的手性片 段，可被进一步氧化为咪唑，作为合成天然产物的底物， 因而具备较高的应用价值.

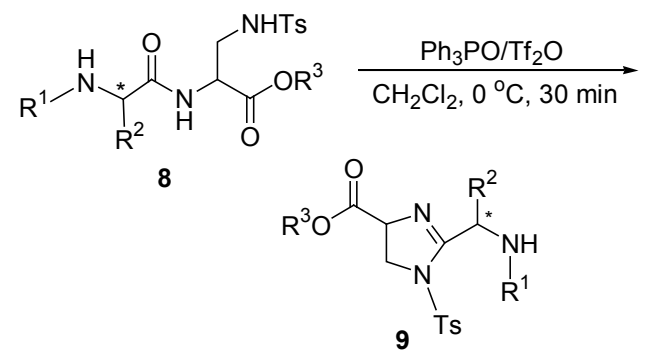

2010 年, Zhu等 ${ }^{[28]}$ 将 $\alpha$-氨基酸 10 转化为具有手性的 二胺 11 后, 与氯化物反应得到亚胺类中间体 12 , 随后 使其经分子内亲核反应而关环得到 2-咪唑啉类化合物 (Scheme 9). 这一转变的完成依赖于中间体中苯胺基的 离去，此合成路线的优势在制备手性 2-咪唑啉时表现突 出.

Kuszpit 等 ${ }^{[29]}$ 通过酰胺与呋丙啶的 Heine 增环作用, 合成了多种取代 2-咪唑啉类化合物(Eq. 8). 反应经历亚 胺酰氯中间体的产生、 $\mathrm{HCl}$ 的释放及 2-咪唑啉类化合物 的生成. 由于产生的 $\mathrm{HCl}$ 气体需要碱性物质的中和, 因 此碱对反应有关键性影响: 例如, 加入少量 $\mathrm{Et}_{3} \mathrm{~N}$ 可以加 快反应进程, 而过量的 $\mathrm{Et}_{3} \mathrm{~N}$ 则阻止反应中间体的生成 从而影响反应产率. 为此, Kuszpit 等经过系列优化后选 定 2,6-二甲基吡啶为碱性试剂. 采用此方法合成咪唑啉 可使呋丙啶的手性完整的转移至产物中.

2011 年, Worrall 等 ${ }^{[30]}$ 利用钯化合物催化得到了 2咪唑啉类化合物. 该小组设计了以化合物 13 为 Münchnone 中间体的合成方法: 将亚胺、酰氯及一氧化 


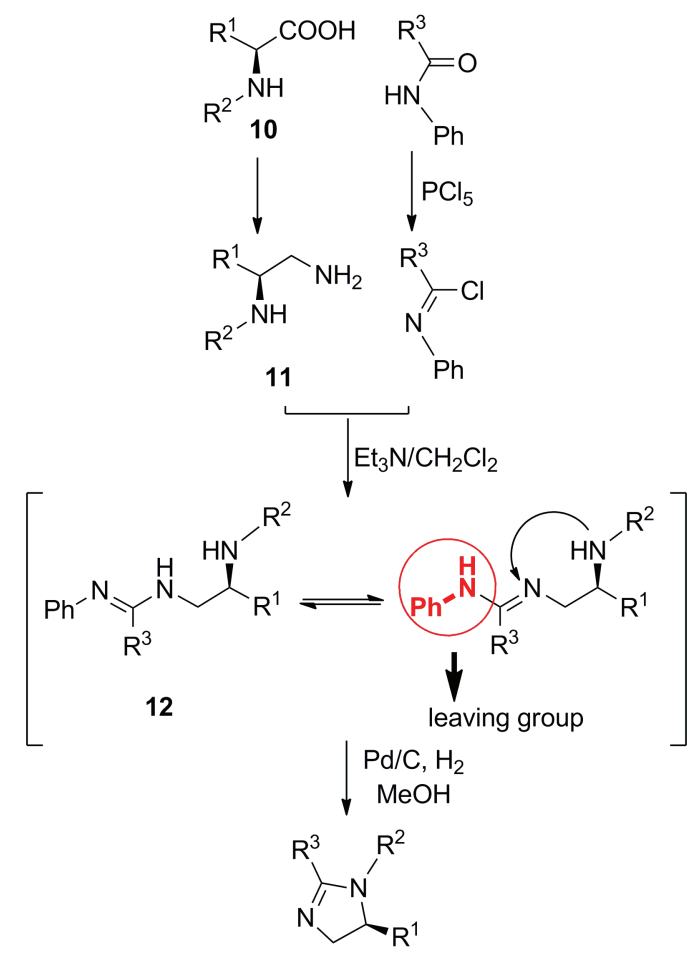

Scheme 9

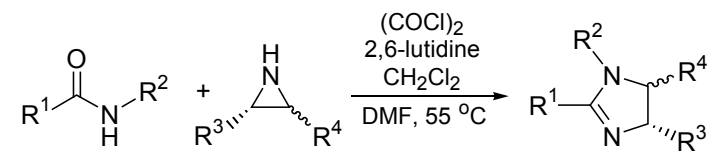

碳混合首先生成中间体, 再与另一分子亚胺作用继而得 到目标产物 15 (Scheme 10).

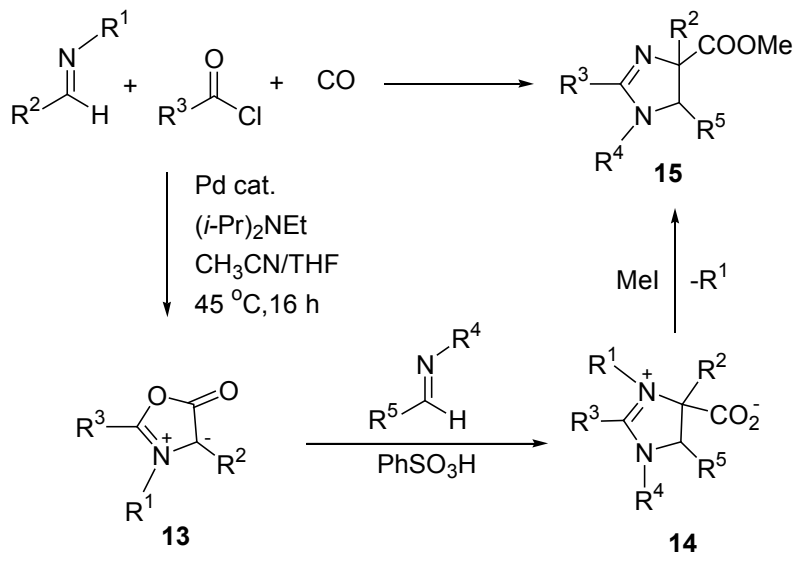

Scheme 10

2012 年, Jiang 等 ${ }^{[31]}$ 设计合成了氟烷基取代的 2-咪 唑啉衍生物, 该方法以取代 1,2-乙二胺及氟烷基羧酸为 原料, 在 $\mathrm{PPh}_{3} / \mathrm{CX}_{4}$ 的催化下完成(Scheme 11). 反应初始 阶段首先生成酰胺 16, 随后其在催化剂 $\mathrm{PPh}_{3} / \mathrm{CX}_{4}$ 的作 用下转变为中间体 17 , 最后通过分子内的关环作用脱 去卤素得到 2-咪唑啉类化合物 18.

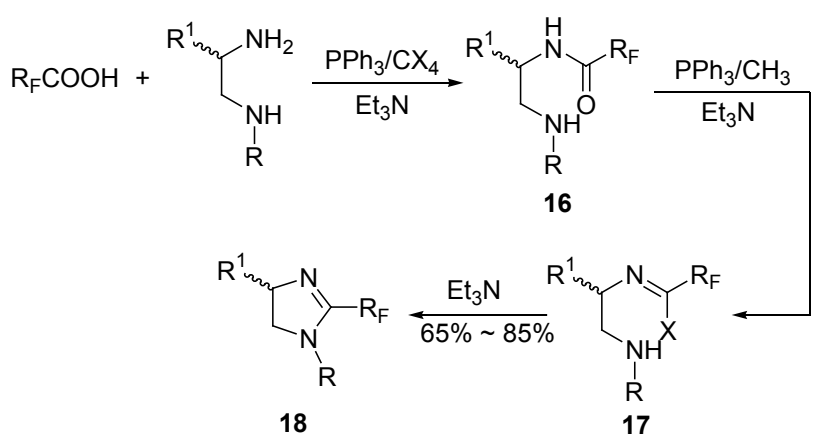

$\mathrm{X}=\mathrm{Cl}, \mathrm{Br}$

$\mathrm{Y}=\mathrm{N} ; \mathrm{R}=\mathrm{Me}, \mathrm{Et}, \mathrm{Ph}, \mathrm{Bn} ; \mathrm{R}^{1}=\mathrm{H}$

$\mathrm{R}_{\mathrm{F}}=\mathrm{CF}_{3}, \mathrm{HCF}_{2}, \mathrm{C}_{3} \mathrm{~F}_{7}, \mathrm{BrCF}_{2}$

\section{Scheme 11}

\section{4 以醛或酮为底物合成}

与前两类方法相比，以醛或酮为底物合成咪唑啉的 方法于近年才被广泛报道.

Jones 等 ${ }^{[32]}$ 于 2003 年在合成化合物 21 时, 通过苯 甲醛与液氨的重排反应意外制得三苯基取代的 2-咪唑 啉 20 (Scheme 12). 反应过程中, 底物首先关环得到 2,4,5-三苯基-3-咪唑啉 19, 而后经碱处理转变为 2-咪唑 啉类化合物 20.

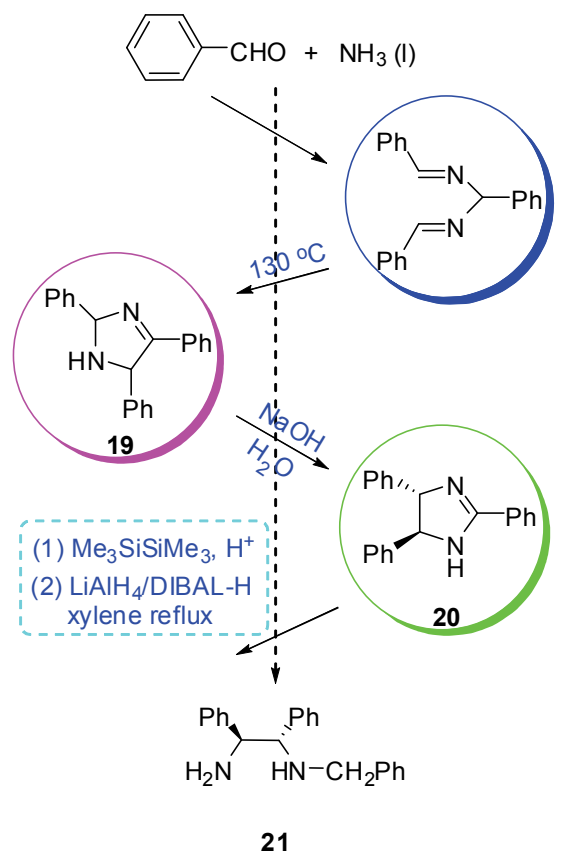

Scheme 12

由于咪唑啉中碳原子的化合价不同于醛中的碳原 子, 因此以醛及二胺为底物合成时均需氧化剂的协助. 2005 年以来, 已有多种氧化醛与乙二胺制备 2-咪唑啉 类化合物方法的报道(Scheme 13).

2005 年, Fujioka 等 ${ }^{[33]}$ 首次报道了醛与乙二胺为底 物制备咪唑啉的方法. 该法以 $\mathrm{NBS}$ 为氧化剂于 $\mathrm{CH}_{2} \mathrm{Cl}_{2}$ 


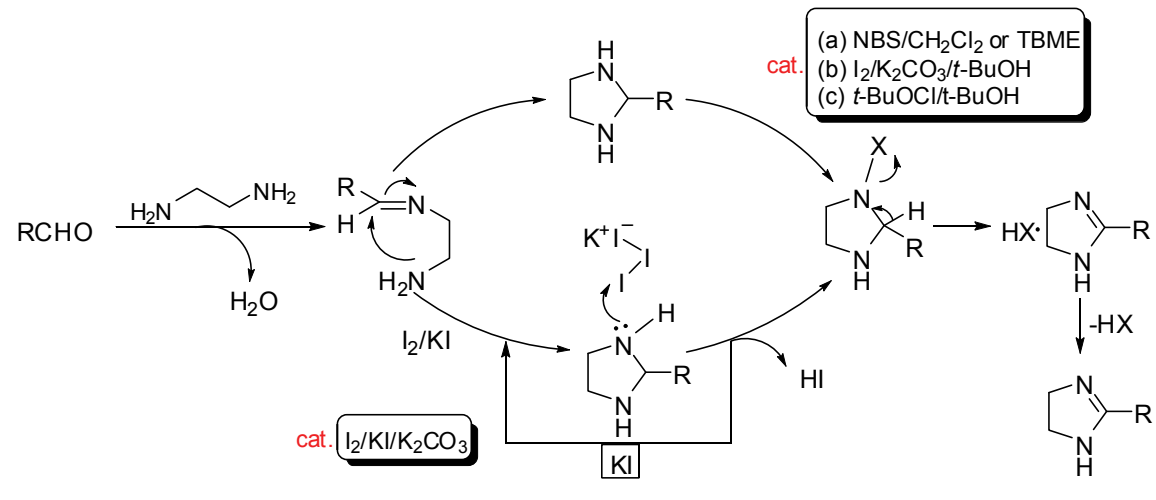

Scheme 13

中进行, 体系中加入适量 $\mathrm{NaOH}$ 或 $\mathrm{NaHCO}_{3}$ 以保证反应 的碱性环境(Scheme 13), 考虑到氯代溶剂多具毒性, Fujioka 对溶剂条件进行了优化 ${ }^{[34]}$, 实验对比了几种较 为常用的有机溶剂, 最终确定以甲基叔丁基醚(TBME) 为反应介质. 采用此方法合成 2-咪唑啉可确保含有氰基 或酯基的醛在转变过程中始终保持官能团的完整性.

2006 年, Gogoi 等 ${ }^{[35]}$ 利用 $\mathrm{I}_{2}$ 的氧化性, 在 KI 及 $\mathrm{K}_{2} \mathrm{CO}_{3}$ 的协助下, 于水溶液中成功地以醛及胺为底物制 备了系列 2-咪唑啉类化合物(Scheme 13). 同年, Ishihara 等 ${ }^{[36]}$ 提出使用 $\mathrm{I}_{2}$ 为氧化剂, 于溶剂 $t-\mathrm{BuOH}$ 中氧化醛及 乙二胺制备 2-味唑啉的方法(Scheme 13). 此外, 还可以 伯醇为合成底物, 只是耗时稍长 ${ }^{[37]}$ (Eq. 9). 2007 年, Ishihara 等 ${ }^{[38]}$ 进一步研究发现, $t-\mathrm{BuOCl}$ 可替代 $\mathrm{I}_{2}$ 起到氧 化作用促使反应顺利进行.

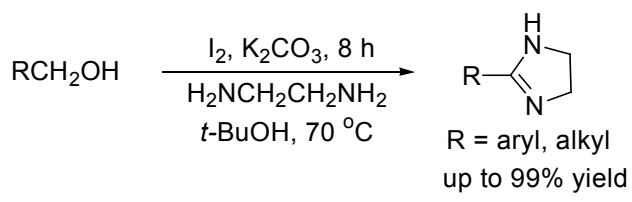

其它廉价的氧化试剂也可用于醛与二胺协同构筑 咪唑啉结构. Sayama ${ }^{[39]}$ 以芳香醛为底物、PHPB 为氧化 剂于水溶液中合成了多种 2-咪唑啉类化合物, 产率理想

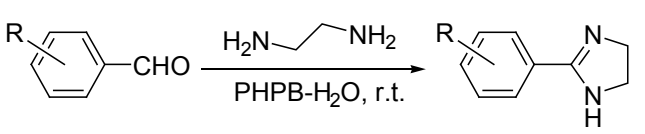

2009 年, Sant Anna 等 ${ }^{[40]}$ 利用超声波提供的能量, 在 无需催化剂的作用下于水溶液中制备了 2-咪唑啉(Eq. 11). 与其它制备法相比, 此合成路线操作简单、反应耗 时短、成本低廉且绿色环保.

$$
\mathrm{R}-\mathrm{CHO}+\mathrm{H}_{2} \mathrm{~N} \simeq \mathrm{NH}_{2} \frac{\left.\left.\mathrm{H}_{2} \mathrm{O}, \mathrm{l}\right)\right), \mathrm{NBS}}{65 \sim 70^{\circ} \mathrm{C}, 12 \sim 18 \mathrm{~min}} \underset{\mathrm{H}}{\mathrm{N}}-\mathrm{R}(11)
$$

2011 年, $\mathrm{Bai}$ 等 ${ }^{[41]}$ 报道了以 $\mathrm{H}_{2} \mathrm{O}_{2}$ 为氧化剂合成 2咪唑啉类化合物的最新方法, 并对反应机理进行了完善 (Scheme 14)。该法以醛及乙二胺为合成底物，在 $\mathrm{NaI}$ 、 无水 $\mathrm{MgSO}_{4}$ 的辅助下完成, 反应产率高且对环境友好.

2011 年, Singh 等 ${ }^{[42]}$ 首次报道了一种以氮杂环环丁 酮衍生物为原料, 合成硫基 2-咪唑啉的简便方法 (Scheme 15). 反应中间体 22 可与多种卤代烷试剂反应, 极大地丰富了产物 2-咪唑啉化合物的种类. 该合成方法 的突出特点是反应过程不使用质子溶剂及 Lewis 酸, 因 此适用范围极广.

2012 年, Murai 等 ${ }^{[43]}$ 以环丁酮类化合物及取代乙二 (Eq. 10).

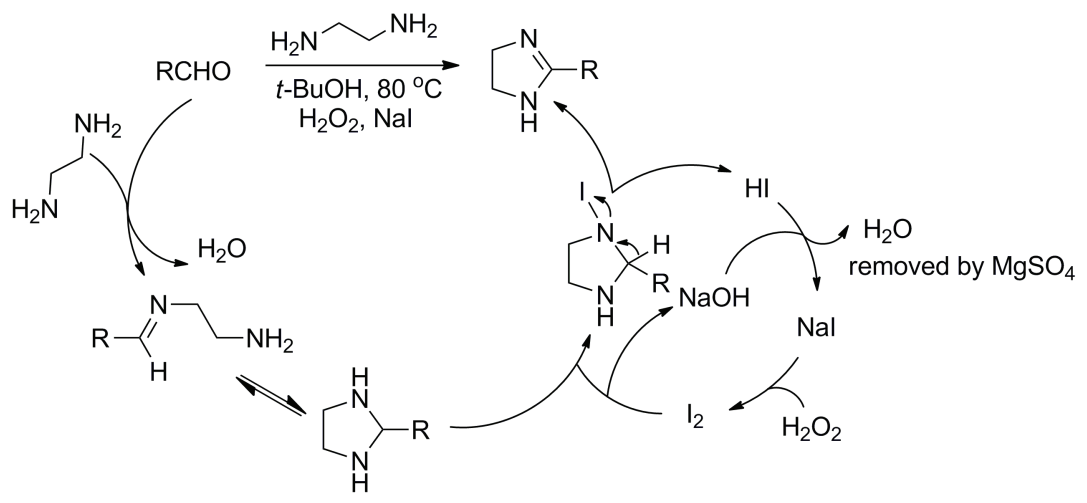

Scheme 14 


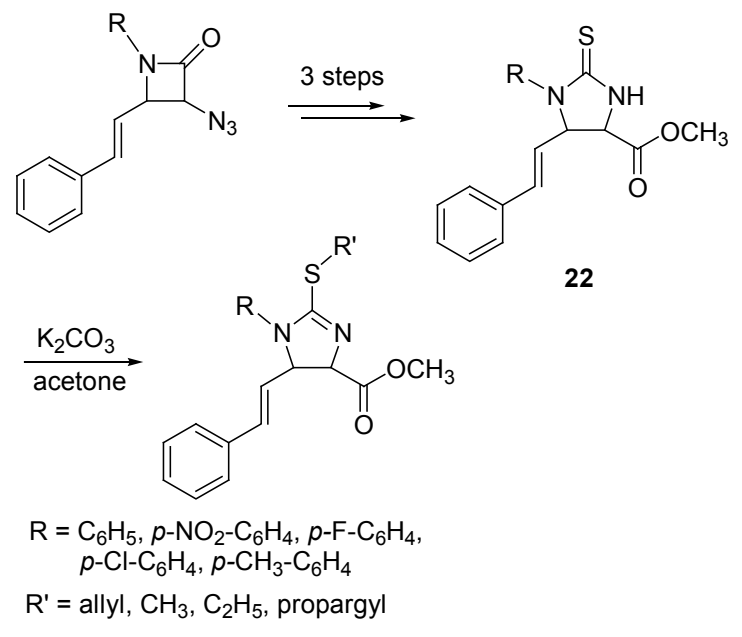

Scheme 15

胺为原料, 通过重排作用制得多种双环结构 2-咪唑啉衍 生物(Scheme 16). 该方法通过缩醛胺中间体的 NBS 氧 化重排实现, 是首例以环酮与二胺为原料的制备方法, 具有开创意义.

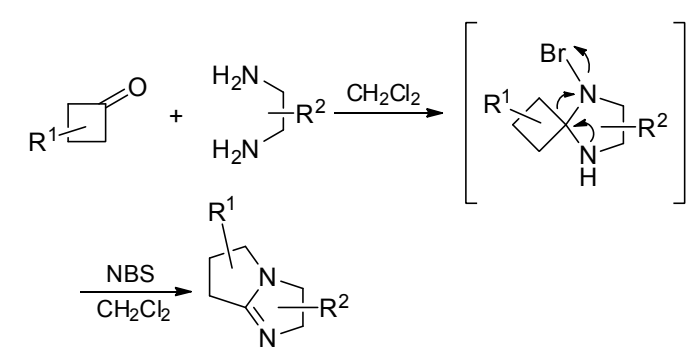

Scheme 16

\section{5 其它底物合成法}

2002 年, Peddibhotla 等 ${ }^{[44,45]}$ 报道了路易斯酸催化惡 唑啉转化合成咪唑啉骨架结构的方法(Eq. 12), 这一转 变遵循 Michael 加成过程而非开环过程(Scheme 17). 反 应底物噁唑啉由 $N$-酰基-氨基酸脱水即可得到, TMSCl 可加速反应的进行, 产物均为纯度较高的非对映异构 体.

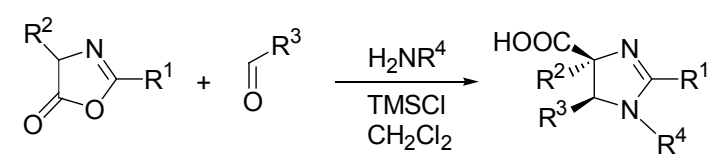

同年, Servi 等 ${ }^{[46,47]}$ 报道了另一种简便有效地合成 2芳胺基-2-咪唑啉 24 的方法(Scheme 18). 该方法所使用 的合成底物芳基取代亚胺 23 由 $\mathrm{CS}_{2}$ 及苯胺混合后经 $\mathrm{CH}_{3} \mathrm{I}$ 处理得到(63\%), 反应物 23 与 1,2 -乙二胺混合, 在 微波协助下几分钟内即可反应完全(产率 83\%).

2004 年, Huh 等 ${ }^{[48]}$ 提出了一种以 2-芳基-1,1-二澳乙

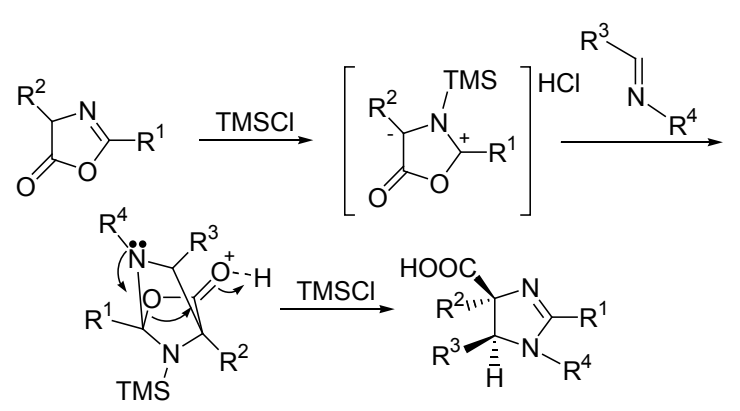

Scheme 17
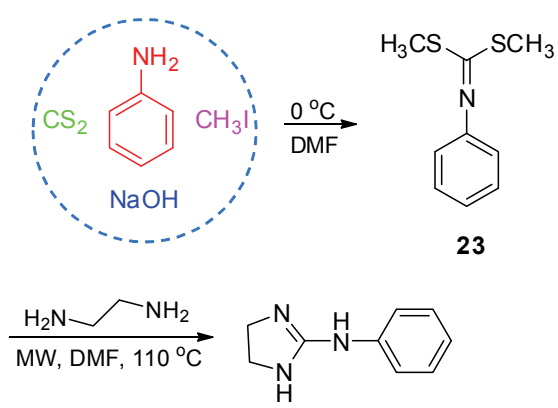

24

\section{Scheme 18}

烯(25)为底物合成 2-咪唑啉 26 的新方法(Scheme 19). 该 路线的优点是反应条件温和且产率稳定, 得到的 2-咪唑 啉可氧化为咪唑及其衍生物，提供了一种简洁的制备具 药物学活性的 2-咪唑啉及咪唑类化合物的路线.

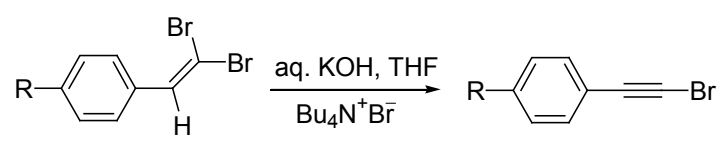

25

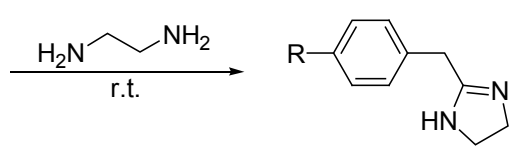

26

Scheme 19

Schmitz 等 ${ }^{[49,50]}$ 于 2005 提出并于 2007 年完善了一 种以醛、腈、胺为反应底物合成咪唑啉的方法(Eq. 13). 该方法遵循 Mannich 反应的基本规则: 首先得到亚胺类 化合物; 随后，亚胺类化合物与腈作用生成中间体；进 而关环得到产物. 溶剂的性质对反应的影响较大，极性 溶剂可促进反应的进程, 甚至不需要催化剂的辅助.

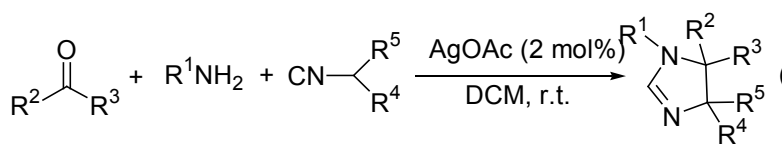

2009 年, Lakner 等 ${ }^{[51]}$ 报道的合成 2-咪唑啉类化合物 
的方法分三步推进(Scheme 20). 底物 27 首先与酰氯作 用生成酰胺 28, 随后分子中的羰基与苯胺发生加成反 应，生成的中间体 29 通过分子内的脱水关环得到三取 代的 2-咪唑啉类化合物 30 (Scheme 20). 采用相同的合 成策略可制得咪唑啉类化合物 31 (Scheme 21), 这两种 咪唑啉均具有很高的稳定性, 可作为生物合成的基本合 成底物.
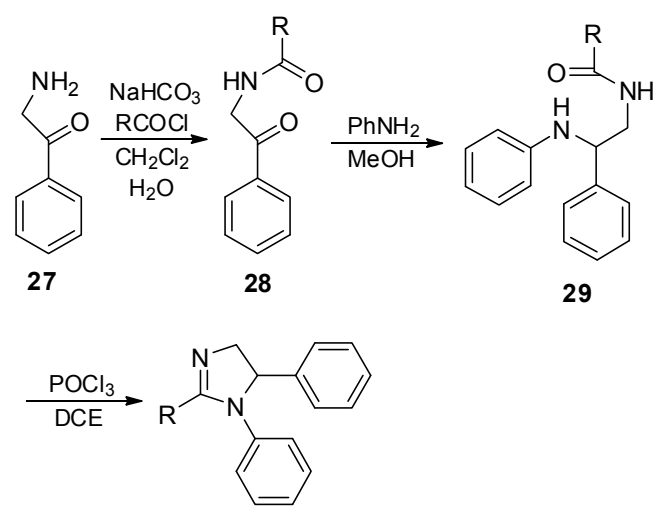

30

Scheme 20<smiles>[R]C(=O)c1cccc(NCC(=O)c2cccc(N(C)N)c2)c1</smiles><smiles>[R]C(=O)N(CC(=O)c1ccccc1)c1ccccc1</smiles>

31

Scheme 21
2011 年, Zhou 等 ${ }^{[52]}$ 报道了一种多组分物质制备咪 唑啉的方法. 此方法以烯烃、腈、胺及 NBS 为底物, 通 过溴离子引发的一系列亲电反应完成转化, 适合于制备 结构复杂的多取代 2-咪唑啉类衍生物(Scheme 22). 反应 过程中，卤素正离子与烯烃结合产生溴鎓离子，这种特 殊的反应方式致使此法具有一定的空间选择性. 随后, Zhou 等 ${ }^{[33]}$ 改变原料组成, 利用此方法成功合成了多种 胍类化合物(Scheme 22).

2012 年, Yamagishi 等 ${ }^{[54]}$ 通过卤代炔烃与单取代 2咪唑啉的亲核加成作用, 使原本结构单一的 2-咪唑啉得 到了丰富，制备过程简单高效且有较好的立体选择性 (Eq. 14).

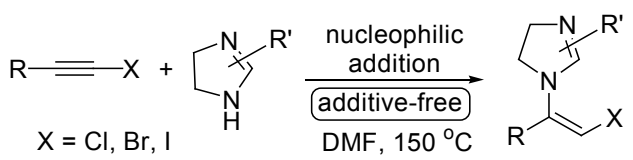

\section{2-咪唑啉类化合物的应用进展}

2-咪唑啉及其衍生物作为药物中间体，主要应用于 抗高血压、抗肿瘤等药物研发, 也大量用作工业领域的 缓蚀剂、有机合成的催化剂等.

\section{1 在医药领域的应用}

2-咪唑啉骨架基优异的生理活性，广泛应用于药物 的设计合成中. 可乐定(Clonidine $)^{[55]}$ 是一种含 2-咪唑啉 基的广谱降血压药，其作用于中枢神经实现降压，但其 毒副作用较大, 大量服用可导致休克、心率缓慢，甚至 可造成呼吸循环衰竭死亡. 第二代中枢性降压药莫索尼 定(Moxonidine)仍然保留 2-咪唑啉骨架基, 仅在苯环上 进行修饰，其药效大为提高，副作用减少，尤其适用于 原发性高血压(Scheme 23).
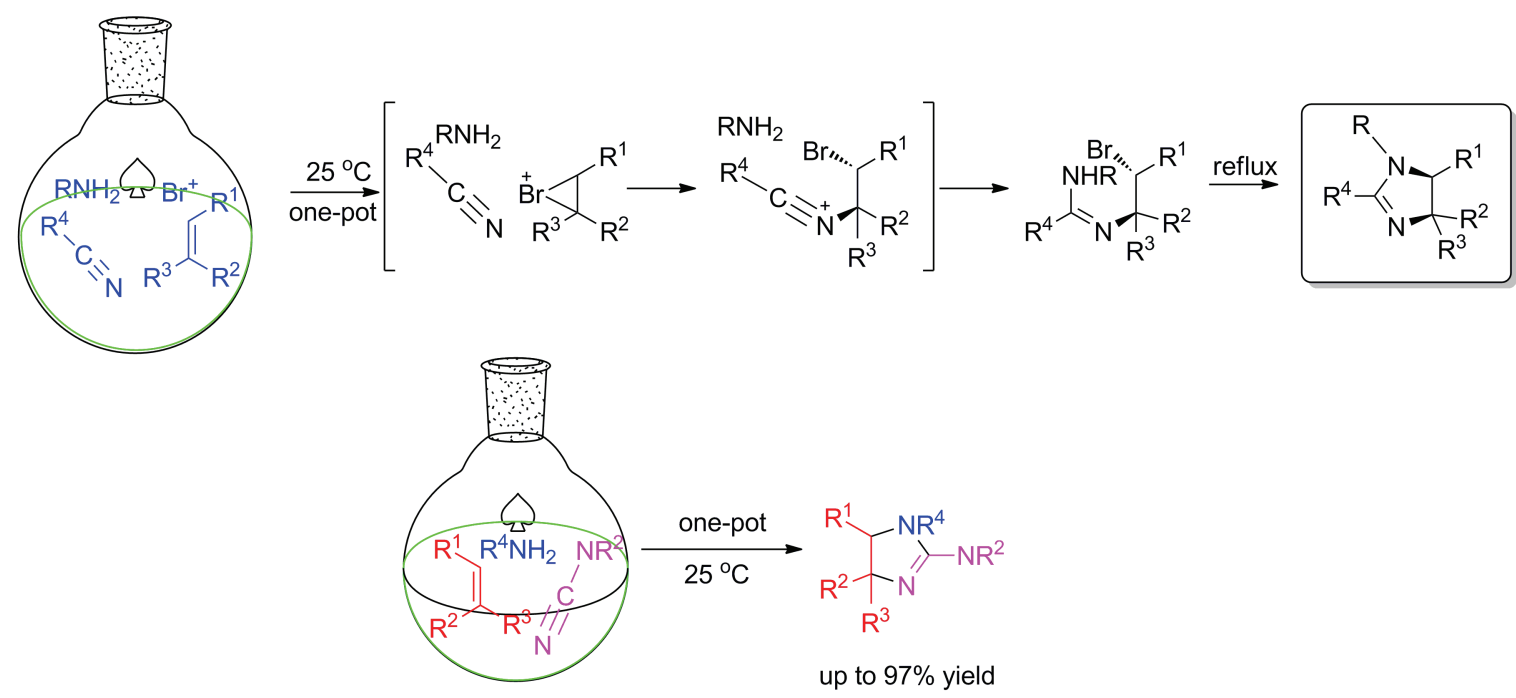

Scheme 22 
<smiles>Clc1cccc(Cl)c1NC1=NCCN1</smiles>

Clonidine<smiles>COc1cc(C)cc(OC)c1NC1=NCCN1</smiles>

Moxonidine

\section{Scheme 23}

$\mathrm{p} 53$ 是为人熟知的抑癌基因, 人类一半以上的癌症 与该基因有关, 使 $\mathrm{p} 53$ 脱离 MDM2 的控制可为癌症治疗 提供新策略 ${ }^{[56,57]}$. 天然产物 Nutlin-3 因 2-咪唑啉活性中 心的存在, 而具有优良的抗肿瘤活性 ${ }^{[58,59]}$. 近年来, 多 种 2-咪唑啉类物质已发展成为 p53-MDM2 结合抑制剂. 2012 年, $\mathrm{Hu}$ 等 ${ }^{[60]}$ 在最新研究中, 设计合成了系列 2-咪坐 啉类衍生物 (Scheme 24), 其中部分化合物可增强 p53-MDM2 蛋白结合抑制效力或抑制细胞增殖, 具有潜 在的抗癌效果.

\section{2 在工业防腐领域的应用}

咪唑啉类缓蚀剂具有无特殊的刺激性气味、热稳定 性好及毒性低等特点 ${ }^{[61]}$. Bajpai ${ }^{[62]}$ 及陶亮亮 ${ }^{[63]}$ 曾分别综 述了这类咪唑啉季铵盐的合成方法及应用进展. 2011 年，樊国栋等 ${ }^{[64]}$ 设计合成了一种新型阳离子咪唑啉季 铵盐缓蚀剂(Eq. 15). 该缓蚀剂具备良好的水溶性及分 散性，极易吸附在金属表面起到缓蚀作用，对钢腐蚀具 有良好的抑制. 当缓蚀剂的浓度维持在 $100 \mathrm{mg} / \mathrm{mL}$ 左右 时，其缓蚀率可达 $90 \%$ 以上，且在酸性环境中效果较佳.

2012 年, 孟邱等 ${ }^{[65]}$ 合成了一种以乙酸为转化剂的 绿色环保型咪唑啉缓蚀剂, 并对其缓蚀性能进行了研究 (Eq. 16). 结果显示, 该缓蚀剂较其它季铵化试剂(如氯 化茮)毒性更低, 水溶性更好且具有物理和化学的双重 吸附作用。

本课题组 ${ }^{[14]}$ 在咪唑啉两性离子、阳离子及非离子表

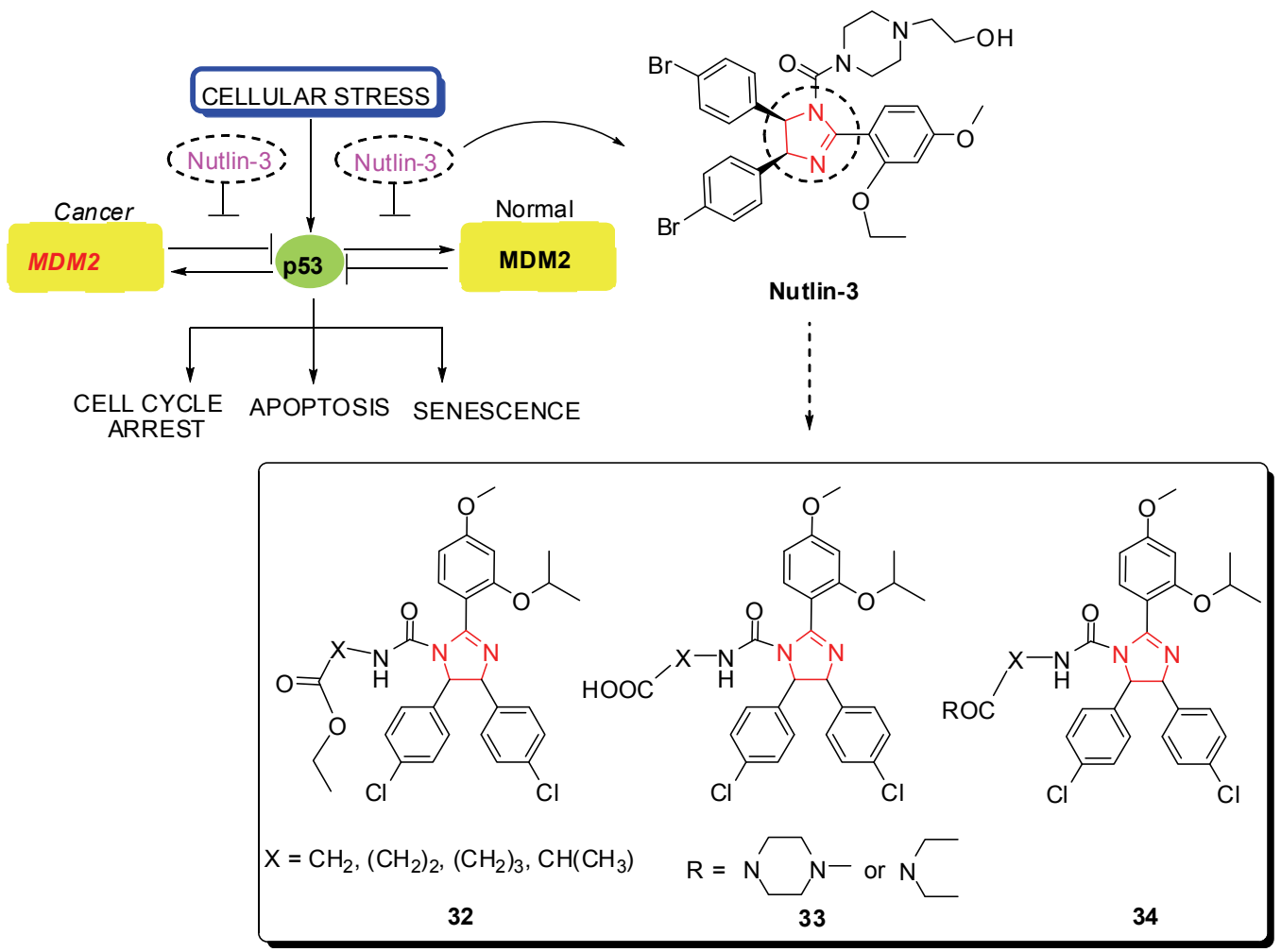

Scheme 24<smiles></smiles>

$\mathrm{H}_{3} \mathrm{C}\left(\mathrm{H}_{2} \mathrm{C}\right)_{7} \mathrm{HC}=\mathrm{CH}\left(\mathrm{CH}_{2}\right)_{7} \mathrm{COOH}+\mathrm{NH}_{2}\left(\mathrm{CH}_{2} \mathrm{CH}_{2} \mathrm{NH}_{2} \mathrm{CH}_{2} \mathrm{CH}_{2} \mathrm{NH}_{2}+\mathrm{CH}_{3} \mathrm{COOH} \stackrel{\text { Heat }}{\longrightarrow} \mathrm{H}_{3} \mathrm{C}\left(\mathrm{H}_{2} \mathrm{C}\right)_{7} \mathrm{HC}=\mathrm{CH}\left(\mathrm{CH}_{2}\right)_{7}\right.$ 
面活性剂方面也有系统的研究, 其中咪唑啉盐阳离子表 面活性剂，其阳离子在金属表面吸附形成覆盖保护膜， 是一种吸附模型缓蚀剂. 最为典型的是 1-氨乙基-2-长 链烷基咪唑啉盐酸盐, 此类缓蚀剂用量少, 缓蚀效果显 著, 咪唑啉环上引入其它活性基团, 可以调控咪唑啉在 金属表面的吸附，从而优化缓蚀性能(Scheme 25).

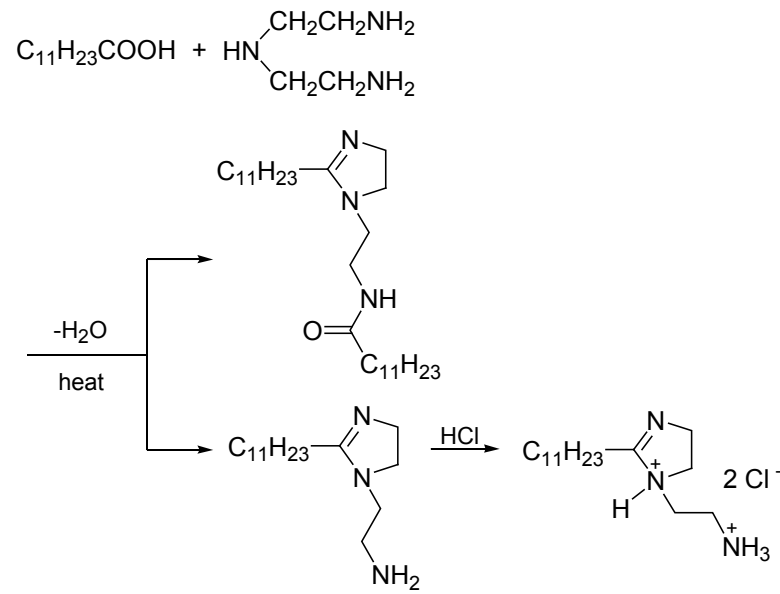

Scheme 25

此外, 功能化的 2-咪唑啉类化合物还被大量用作离 子液体成为对环境友好的 “绿色溶剂”, 2012 年初, Tang 等 ${ }^{[66]}$ 简述了离子液体领域的最新研究进展.

\section{3 在有机合成领域的应用}

\subsection{1 在催化合成方面的应用}

有机配体在新型催化剂骨架的设计和构建中具有 重要的作用, 咪唑啉及其衍生物作为催化配体应用于有 机合成中开创了多个催化反应的新领域, 如 2-咪唑啉与 钯配位后可作为 Heck 反应的有效催化 ${ }^{\text {剂 }}{ }^{[67]}$ (Scheme 26).

1989 年, Bottegh 等 ${ }^{[68]}$ 首先报道合成了双配位的一 元咪唑啉配体 35 37 (Scheme 27), 首次将手性咪唑啉 配体应用于催化合成. 虽然催化产率仅为 $5.2 \%$, 但研究 工作极富开创性.

2011 年, Liu 等 ${ }^{[69]}$ 合成出一种具有刚性结构的咪唑 啉盐, 并意外地发现其在催化酰胺类物质的分子内 $\alpha$-烷 基化反应时表现出很好催化活性(Eq. 17), 催化剂结构 中的 NHC 刚性环状片段对其催化活性及产物的立体选 择有关键性影响.

2012 年, Yuan 等 ${ }^{[70]}$ 制备的系列手性咪唑啉类化合 物可与锌配位后用作 Mannich 反应的催化剂(Eq. 18). 该催化剂的添加不仅可使反应在温和条件下进行, 还具 有较高的催化效率.

2012 年, De la Fuente 等 ${ }^{[711}$ 将合成得到的 2-咪唑啉 衍生物 40 与 $\left[\mathrm{Pd}\left(\eta^{3}-\mathrm{C}_{3} \mathrm{H}_{5}\right) \mathrm{Cl}\right]_{2}$ 配合后, 以咪唑离子液体
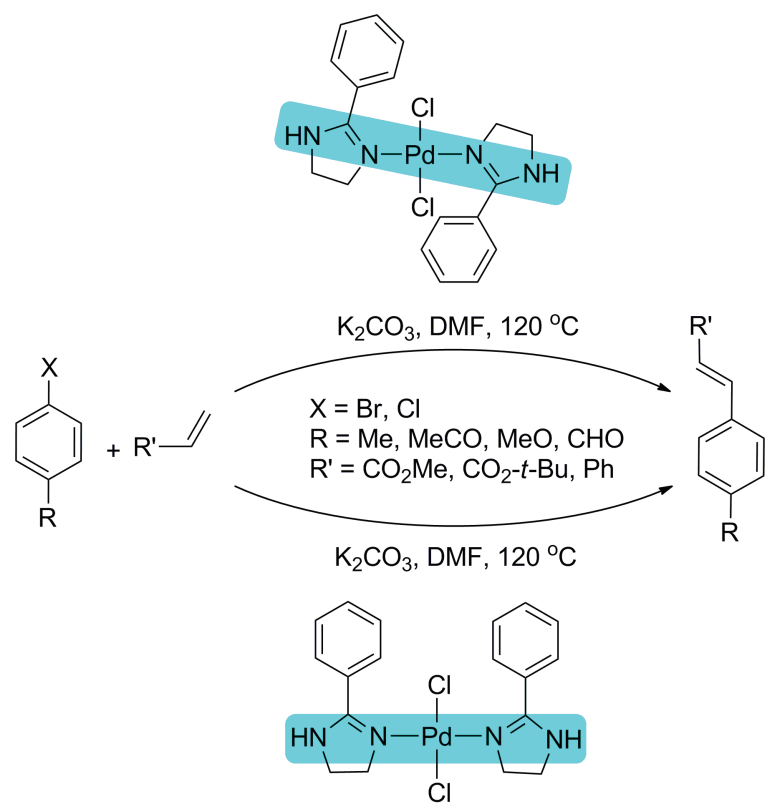

Scheme 26

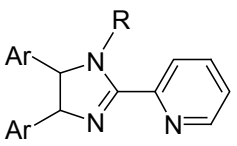

$\mathrm{R}=\mathrm{H}, \mathrm{Bn}, \mathrm{Me}, \mathrm{Ts}, \mathrm{T}$

35

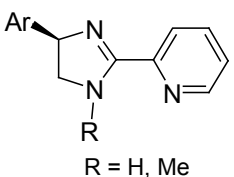

36

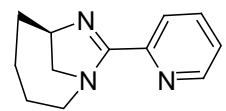

37
Scheme 27

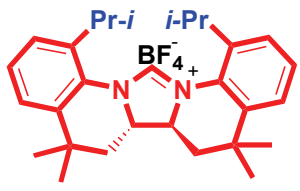

38
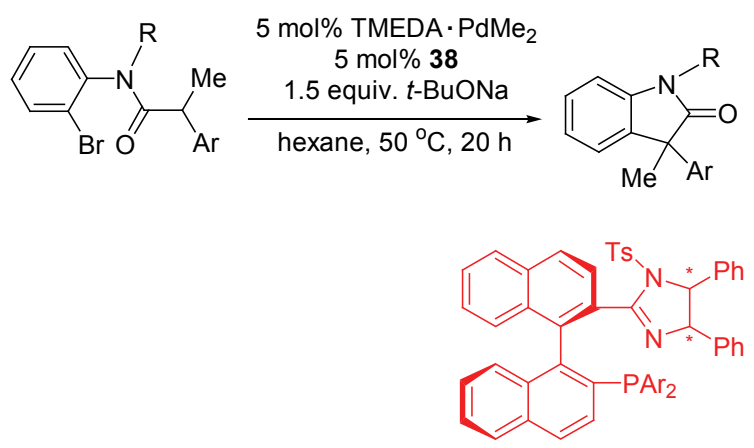

39

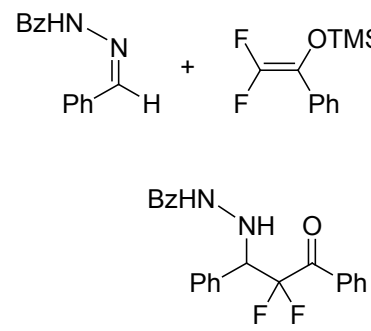


41 为溶剂, 高效率实现了化合物 42 的烯丙基烷烃化反 应(Eq. 19). 此报道充分展现了 2-咪唑啉类化合物及功 能化的咪唑啉的巨大研究价值及广阔应用领域.
2.3.2 在仿生有机合成方面的应用。

生命活动和谐进行, 酶在其中起着十分重要的作 用，咪唑啉及其衍生物仿四氢叶酸辅酶一碳单元转移反
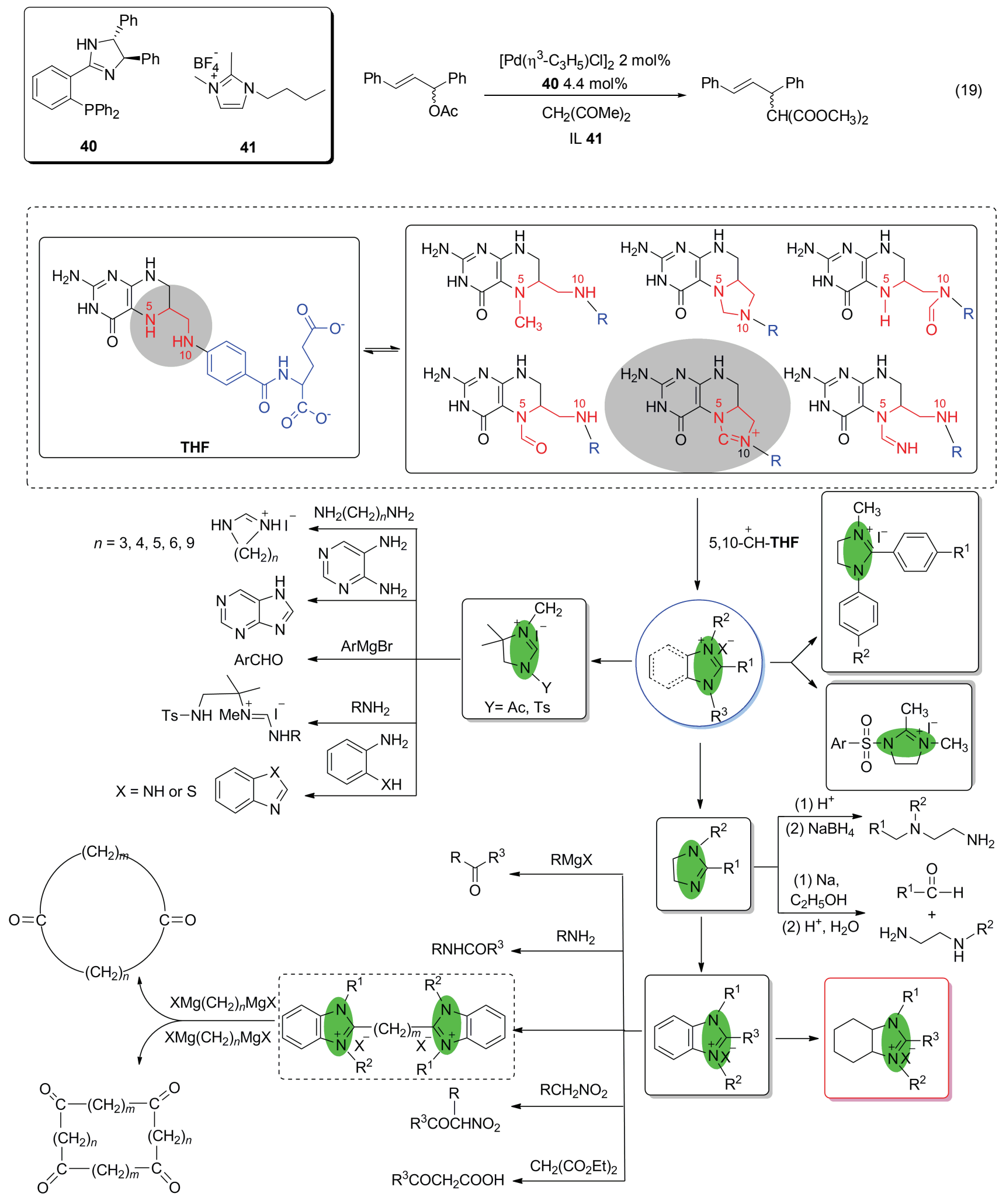

Scheme 28 
应是仿生有机合成化学研究的重要课题 ${ }^{[72]}$. Pandit 等 ${ }^{[73,74]}$ 曾在 1979 年最早提出以碘化 3-甲基-1-对苯磺酰 基(或乙酰基)咪唑啉作为甲酸氧化态的四氢叶酸辅酶模 型化合物, 并研究了该模型与各种亲核试剂的反应; 夏 炽中等 ${ }^{[75 ~ 77]}$ 在此基础上, 进一步做了大量开创性工作, 使咪唑啉模型化合物仿四氢叶酸辅酶生物学功能的合 成研究较为系统.

本课题组在咪唑啉化学研究基础上, 提出芳香苯并 咪唑啉类化合物, 即四氢叶酸辅酶另一新型系列模 型——苯并咪唑及衍生物, 并研究和报道了该模型化合 物与多种亲核试剂的加成一水解反应, 实现了一碳单元 转移的仿生合成, 为多种醛、酮和大环酮等有机化合物 提供了常规方法难以实现或实现不理想的合成新方 法(Scheme 28) ${ }^{[78]}$. 在此基础上, 进一步研究去芳香结构 的环己烷并咪唑啉系列模型物——六氢苯并咪唑及其 衍生物, 探索其在四氢叶酸辅酶仿生化学中的反应行 为.

\section{3 小结}

综上可见，2-咪唑啉及其衍生物作为含氮杂环化合 物中的一类重要物质, 具有良好的药物活性且被广泛用 作缓蚀剂等, 更是不对称催化反应重要的催化剂配体功 能调控基元片段. 近年来, 过渡金属配合物催化剂的应 用极大地促进了咪唑啉类化合物合成方法的发展，而多 组分串联反应在 2-咪唑啉类化合物骨架的构建上突显 其合成的灵活性. 随着人们对新型药物、性能更优的缓 蚀剂及催化剂的需求, 高效、经济、绿色的 2-咪唑啉合 成方法的探索仍将是今后研究的热点. 相信随着研究的 深入, 2-咪唑啉及其衍生物也将有更广阔的应用前景.

\section{References}

[1] Grimmett, M. R. In Comprehensive Heterocyclic Chemistry, Vol. 3, Eds.: Katrizky, A. R.; Rees, C. W., Pergamon, Oxford, 1996, p. 77.

[2] Crouch, R. D. Tetrahedron 2009, 65, 2387.

[3] Liu, H.; Du, D.-M. Adv. Synth. Catal. 2009, 351, 489.

[4] Oxley, P.; Short, W. F. J. Chem. Soc. 1947, 497.

[5] Levesque, G.; Gressier, J.-C.; Proust, M. Synthesis 1981, 963.

[6] Dash, P.; Kudav, D. P.; Parihar, J. A. J. Chem. Res. 2004, 490.

[7] Mirkhani, V.; Moghadam, M.; Tangestaninejada, S.; Kargar, H. Tetrahedron Lett. 2006, 47, 2129.

[8] Mirkhani, V.; Mohammadpoor-Baltork, I.; Moghadam, M.; Tangestaninejad, S.; Abdollahi-Alibeik, M.; Kargar, M. Appl. Catal. A: Gen. 2007, 325, 99.

[9] Mohammadpoor-Baltork, I.; Mirkhani, V.; Moghadam, M.; Tangestaninejad, S.; Zolfigol, M. A.; Abdollahi-Alibeik, M.; Khosropour, A. R.; Kargar, H.; Hojati, S. F. Catal. Commun. 2008, 9, 894.

[10] Mohammadpoor-Baltork, I.; Moghadam, M.; Tangestaninejad, S.; Mirkhani, V.; Hojati, S. F. Polyhedron 2008, 27, 750.

[11] Shaabani, A; Seyyedhamzeh, M.; Maleki, A.; Rezazadeh, F. Appl. Catal. A: Gen. 2009, 358, 146.
[12] Nasr-Esfahani, M.; Montazerozohori, M.; Moghadam, M.; Akhlaghi, P. ARKIVOC 2010, 2, 97.

[13] Nasr-Esfahani, M.; Montazerozohori, M.; Mehrizi, S. J. Heterocycl. Chem. 2011, 48, 249

[14] Shi, Z. Selected Topics on Modern Heterocyclic Chemistry, Shaanxi Science and Technology Press, Xi'an, 1998, p. 55, p. 211 (in Chinese)

(史真, 现代杂环化学选论, 陕西科学技术出版社, 西安, 1998 , p. 55, p. 211.)

[15] Zhang, J.; Wang, X.; Yang, M.-P.; Wan, K.-R.; Yin, B.; Wang, Y.-X.; Li, J.-L.; Shi, Z. Tetrahedron Lett. 2011, 52, 1578.

[16] Li, X.-N.; Zhou, B.-Y.; Zhang, J.; She, M.-Y.; An, S.-J.; Ge, H.-X.; Li, C.; Yin, B.; Li, J.-L.; Shi, Z. Eur. J. Org. Chem. 2012, 1626

[17] Hiyama, T.; Koide, H.; Fujita, S.; Nozaki, H. Tetrahedron 1973, 29 , 3137.

[18] Concellón, J. M.; Riego, E.; Suárez, J. R.; García-Granda, S.; Díaz, M. R. Org. Lett. 2004, 6, 4499.

[19] Gandhi, S.; Bisai, A.; Prasad, B. A. B.; Singh, V. K. J. Org. Chem. 2007, 72, 2133.

[20] Li, X.; Yang, X.-Q.; Chang, H.-H.; Li, Y-W.; Ni, B.; Wei, W.-L. Eur. J. Org. Chem. 2011, 3122.

[21] Wei, W.-L.; Yang, X.-Q.; Li, X.; Chang, H.-H. Chem. Ind. Times 2011, 23, 44 (in Chinese). (魏文珑, 杨学绮, 李兴, 常宏宏, 化工时刊, 2011, 23, 44.)

[22] Benito-Garagorri, D.; Bocokić, V.; Kirchner, K. Tetrahedron Lett. 2006, 47, 8641 .

[23] Nakamura, S.; Maeno, Y.; Ohara, M.; Yamamura, A.; Funahashi, Y.; Shibata, N. Org. Lett. 2012, 14, 2960.

[24] Neef, G.; Eder, U.; Sauer, G. J. Org. Chem. 1981, 46, 2824.

[25] Aleksandrov, A. A.; El'chaninov, M. M. Russ. J. Appl. Chem. 2009, $82,2161$.

[26] Boland, N. A.; Casey, M.; Hynes, S. J.; Matthews, J. W.; Smyth, M. P. J. Org. Chem. 2002, 67, 3919.

[27] You, S.-L.; Kelly, J. W. Org. Lett. 2004, 6, 1681.

[28] Zhu, Q.; Lu, Y-X. Org. Lett. 2010, 12, 4156.

[29] Kuszpit, M. R.; Wulff, W. D.; Tepe J. J. J. Org. Chem. 2011, 76, 2913.

[30] Worrall, K.; Xu, B-R.; Bontemps, S.; Arndtsen B. A. J. Org. Chem. 2011, 76, 170.

[31] Jiang, H.; Sun, L.; Yuan, S.; Lu, W.; Wan, W.; Zhu, S.; Hao, J. Tetrahedron 2012, 68, 2858.

[32] Jones, R. C. F.; Hirst, S. C. ARKIVOC 2003, (ii), 133.

[33] Fujioka, H.; Murai, K.; Ohba, Y.; Hiramatsu, A.; Kita, Y. Tetrahedron Lett. 2005, 46, 2197.

[34] Fujioka, H.; Murai, K.; Kubo, O.; Ohba, Y.; Kita, Y. Tetrahedron 2007, 63, 638.

[35] Gogoi, P.; Konwar, D. Tetrahedron Lett. 2006, 47, 79.

[36] Ishihara, M.; Togo, H. Synlett 2006, 227.

[37] Ishihara, M.; Togo, H. Tetrahedron 2007, 63, 1474.

[38] Ishihara, M.; Togo, H. Synthesis 2007, 1939.

[39] Sayama, S. Synlett 2006, 1479.

[40] Sant Anna, G.; Machado, P.; Sauzem, P.; Rosa, F. A.; Rubin,.M. A.; Ferreira, J.; Bonacorso, H.G.; Zanatta, N.; Martins, M. A. P. Bioorg. Med. Chem. Lett. 2009, 19, 546.

[41] Bai, G.-Y.; Xu, K.; Chen, G.-Y.; Yang, Y.-H; Li, T.-Y. Synthesis 2011, 1599.

[42] Singh, P.; Mehra, V.; Anand, A.; Kumar, V.; Mahajan, M. P. Tetrahedron Lett. 2011, 52, 5060.

[43] Murai, K.; Komatsu, H.; Nagao, R.; Fujioka, H. Org. Lett. 2012, 14, 772

[44] Peddibhotla, S.; Jayakumar, S.; Tepe, J. J. Org. Lett. 2002, 4, 3533.

[45] Peddibhotla, S.; Tepe, J. J. Synthesis 2003, 1433. 
[46] Servi, S. S. Afr. J. Chem. 2002, 55, 119.

[47] Genc, M.; Servi, S. Heteroat. Chem. 2005, 16, 142.

[48] Huh, D. H.; Ryu, H.; Kim, Y. G. Tetrahedron 2004, 60, 9857.

[49] Bon, R. S.; B. Van Vliet, B.; Sprenkels, N. E.; Schmitz, R. F.; De Kanter, F. J. J.; Stevens, C. V.; Swart, M.; Bickelhaupt, F. M.; Groen, M. B.; Orru, R. V. A. J. Org. Chem. 2005, 70, 3542.

[50] Elders, N.; Schmitz, R. F.; Kanter, F. J. J.; Ruijter, E.; Groen, M. B.; Orru, R. V. A. J. Org. Chem. 2007, 72, 6135.

[51] Lakner, F. J.; Parker, M. A.; Rogovoy, B.; Khvat, A.; Ivachtchenko, A. Synthesis 2009, 1987.

[52] Zhou L.; Zhou J.; Tan C. K.; Chen J.; Yeung Y-Y. Org. Lett. 2011, $13,2448$.

[53] Zhou, L.; Chen, J.; Zhou, J.; Yeung, Y.-Y. Org. Lett. 2011, 13, 5804 .

[54] Yamagishi, M.; Okazaki, J.; Nishigai, K.; Hata, T.; Urabe, H. Org. Lett. 2012, 14, 34.

[55] Sved, A. F. Eur. J. Pharmacol. 1985, 109, 111

[56] Chari, N. S.; Pinaire, N. L.; Thorpe, L.; Medeiros, L. J.; Routbort, M. J.; McDonnell, T. J. Apoptosis 2009, 14, 336.

[57] Brooks, C. L.; Gu, W. Mol. Cell 2006, 21, 307.

[58] Vassilev, L. T.; Vu, B. T.; Graves, B.; Carvajal, D.; Podlaski, F.; Filipovic, Z.; Kong, N.; Kammlott, U.; Lukacs, C.; Klein, C.; Fotouhi, N.; Liu, E. A. Science 2004, 303, 844.

[59] Harris, C. C. Proc. Natl. Acad. Soc. U. S. A. 2006, 103, 1659.

[60] Hu, C.-Q..; Dou, X.-X.; Wu, Y.-Z.; Zhang, L.; Hu, Y.-Z. Bioorg. Med. Chem. 2012, 20, 1417.

[61] Zhu, L.; Yu, P.; Luo, Y.-B. Mater. Protect. 2003, 36, 12 (in Chinese).

(朱镭, 于萍, 罗运柏, 材料保护, 2003, 36, 12.)

[62] Bajpai, D.; Tyagi, V. K. J. Oleo Sci. 2006, 55, 319.

[63] Tao, L.-L.; Zhu, Y.; Fang, L.-Y.; Hu, R. Leath. Chem. 2011, 28, 30 (in Chinese).
(陶亮亮, 朱燕, 方李艳, 胡瑞, 皮革与化工, 2011, 28, 30.)

[64] Fan, G.-D.; Ge, J.; Cai, L.-L. Spec. Petrochem. 2011, 28, 73 (in Chinese).

(樊国栋, 葛君, 柴玲玲, 精细石油化工, 2011, 28, 73.)

[65] Meng, Q.; Yan, H.; Huang, W.; Cong, Y.-F. Chem. Ind. Eng. 2012, 29, 28 (in Chinese).

(孟邱, 闰慧, 黄玮, 丛玉凤, 化学工业与工程, 2012, 29, 28.)

[66] Tang, B. K.; Bi, W. T.; Tian, M. L.; Row, K. H. J. Chromatogr. B 2012, 904, 1.

[67] Kawamura, K.; Haneda, S.; Gan, Z.; Eda, K.; Hayashi, M. Organometallics 2008, 27, 3748.

[68] Botteghi, C.; Schionato, A.; Chelucci, G.; Brunner, H.; Kürzinger, A.; Obermann, U. J. Organomet. Chem. 1989, 370, 17.

[69] Liu, L-T.; Ishida, N.; Ashida, S.; Murakami, M. Org. Lett. 2011, $13,1666$.

[70] Yuan, Z.-L.; Mei, L.-Y.; Wei, Y.; Shi, M.; Kattamuri, P. V.; McDowell, P.; Li, G.-G. Org. Biomol. Chem. 2012, 10, 2509.

[71] De la Fuente, V.; Fleury-Bregeot, N.; Castillon, S.; Claver, C. Green Chem. 2012, 14, 2715.

[72] Choi, H.; Kwak, M.; Song, H. Synth. Commun. 1997, 27, 1273.

[73] Pandit, U. K.; Bieraugel, H. J. Chem. Soc., Chem. Commun. 1979, 117.

[74] Pandit, U. K. Pure Appl. Chem. 1994, 66, 759.

[75] Xia, C.-Z.; Chen, J.-X.; Zhao, B.-J. Synth. Commun. 2002, 32, 1129.

[76] Zhou, P-W.; Zhao. B.-J.; Chen, J.-X. Heterocycles 2000, 53, 433.

[77] Li, D.-H.; Zhang, Y.-B.; Xia, C.-Z. Heterocycles 2005, 65, 2893.

[78] (a) Jiang, J.-L.; Shi, Z. Synth. Commun. 1998, $28,4137$.

(b) Bai, Y.-J.; Lu, J.; Shi, Z.; Yang, B.-Q. Synlett 2001, 544.

(c) Li, J.-L. Ph.D. Dissertation, Northwest University, Xi'an, 2007 (in Chinese).

(李剑利, 博士论文, 西北大学, 西安, 2007.) 